\title{
Magnetic Properties of cis- and trans-Polyacetylene as Studied by Electron Spin Resonance
}

\author{
P. BERNIER and M. ROLLAND
}

Groupe de Dynamique des Phases Condensées*

C. Linaya, M. Disi, J. Sledz, F. Schue, J. M. FABRE, and L. GIRAL

Laboratoire de Chimie Macromoléculaire, Laboratoire de Chimie Structurale Organique, Université des Sciences et Techniques du Languedoc, Place Eugène Bataillon, 34060 Montpellier-France.

(Received May 27, 1980)

\begin{abstract}
Polyacetylene films strongly react with air, when conserved at room temperature and atmospheric pressure. We present here an experimental electron spin resonance (ESR) study on the variation of the linewidth and number of spins with time and temperature of $c i s-$ and $\operatorname{trans}-(\mathrm{CH})_{x}$ films of different thicknesses. It is shown that this evolution is qualitatively and quantitatively different for the two isomers. The number of resonant spins increases with time with a characteristic time of the order of 8 hours in the case of the cis isomer. For the trans isomer, the number of resonant spins, which is an order of magnitude greater than the final value of the cis isomer, is remarkably constant with time while the linewidth and amplitude are not. In the light of these results, the origin and nature of the observed resonant spins are discussed.
\end{abstract}
KEY WORDS
Isomerization
Magnetic Resonance / Polyacetylene / Oxidation

Polyacetylene is now a material of considerable interest as, upon doping with chemical species as $\mathrm{I}_{2}$, $\mathrm{AsF}_{5}, \mathrm{SbF}_{5}$, etc., its electronic transport properties cover the whole range from insulators to metals and semiconductors. $^{1-3}$ This behaviour is strongly correlated to the intrinsic nature of the polymeric chain which consists of conjugated double bonds in the cis or trans configurations. As a consequence of this nature, polyacetylene is also very sensitive to oxygen which can considerably change its mechanical, ${ }^{4}$ electrical, ${ }^{5}$ and magnetic properties. ${ }^{6,7}$ Such a fact leds to a complicated situation if one tries to interpret quantitatively the properties of doped systems, as the final measured quantities may or may not strongly depend on the whole history of the sample and particularly the presence of air during treatment. In that sense, it is interesting to investigate the behaviour of undoped polyacetylene

\footnotetext{
* Laboratoire associé au C.N.R.S., France.
}

samples in order to determine precisely which type of evolution applies to a given situation.

It is also of interest to investigate the properties of undoped polyacetylene so as to understand the origin of the unpaired electrons which contribute to the observed electron paramagnetic resonance (EPR) signal. For this purpose, the cis and trans isomers cannot be considered as equivalent. Different authors ${ }^{7,8}$ have postulated the existence of neutral defects in the trans isomer, appearing as domain walls or solitons.

This is compatible with the fact that this isomer is energetically unaffected by the interchange of double and single bonds (I),<smiles>C=C=CC=CC=CC=CC=C</smiles> 
But this is not the case for the cis-isomer since cistransoïd and trans-cisoïd are not energetically equivalent ${ }^{9,10}$ (II).<smiles>C/C=C\C=C/C=C\C</smiles>

cis-transoïd<smiles>C=C/C=C\C=C/C=C\C</smiles>

trans-cisoïd
This situation normally allows us to predict different behaviours for the two isomers. In fact, this is not generally qualitatively the case at room temperature as is seen from electrical ${ }^{11}$ and magnetic $^{2,6,7}$ measurements on doped samples. We thus have to make the assumption that in addition to the normal thermal transformation from cis to trans, any doping process induces some change in the cis configuration, as suggested by Chiang et al. ${ }^{11}$

We present here a systematic study, with the evolution of time and temperature and with and without the presence of air, of the magnetic properties of the unstable $c i s-(\mathrm{CH})_{x}$ isomer and the stable trans isomer, using EPR technics. We show that, starting from the low polymerization temperature $\left(-78^{\circ} \mathrm{C}\right)$ at which no unpaired electrons exist on the polymer chains, heating of the samples or/and contact with air causes such spin states to come about. We discuss the origin of this behaviour.

\section{EXPERIMENTAL}

Polyacetylene films were obtained by the method described by Ito et al., ${ }^{12}$ using the $\mathrm{Ti}\left(\mathrm{OC}_{4} \mathrm{H}_{9}\right)_{4}+$ $\mathrm{Al}\left(\mathrm{C}_{2} \mathrm{H}_{5}\right)_{3}$ homogeneous catalytic system in toluene (with molar ratio of $\mathrm{Al} / \mathrm{Ti}=4$ ), at $-78^{\circ} \mathrm{C}$. Only the films growing on the vertical surfaces, coated with the catalytic system, of the polymerization reactor are considered in this study. The thickness varied from 20 to $130 \mu \mathrm{m}$ depending on the time of polymerization.

The as-prepared samples, carefully dried, were conserved under vacuum at $-25^{\circ} \mathrm{C}$. The cis-rich isomer was observed to be stable with time. Isomerization to the trans configuration was performed at $200^{\circ} \mathrm{C}$. The glass tubes containing the samples under vacuum were directly immersed in isopentane for given time ( 5 minutes to 2 hours).

One of the samples was directly polymerized at $-78^{\circ} \mathrm{C}$ inside the EPR tube. The tube was sealed under vacuum the sample being always kept at low temperature. The sample was then immediately introduced in a variable temperature cryostat, stabilized at $-78^{\circ} \mathrm{C}$, inside the spectrometer cavity.

The electron spin resonance (ESR) spectra were recorded on an ER10 Bruker spectrometer working at $9.0 \mathrm{GHz}$. The maximum hyperfrequency power was $30 \mathrm{~mW}$. To study the evolution with time in the presence of air, the sample tube was only open at a specified time and the characteristics of the ESR spectra versus time were followed until a reproducible behaviour was reached.

All the experiments described here were performed two or three times on different samples prepared at different times to be sure that the observed behaviour was not just a spurious effect.

\section{EXPERIMENTAL RESULTS}

\section{The cis-Isomer}

If observed at the polymerization temperature $-78^{\circ} \mathrm{C}$ the ESR spectrum as described previously ${ }^{13}$ showed no signal from the polyacetylene film while a very huge and broad signal centered near $g=1.95$ came from the catalytic system. Such a catalyst signal has been studied by different authors ${ }^{14,15}$ and a strong dependence of its shape with the molar ratio of $\mathrm{A} 1 / \mathrm{Ti}$ was pointed out. Our results are consistent with what is expected for a molar ratio of 4.

Table I. Characteristics of the ESR Lorentzian spectra at room temperature obtained on cis- $(\mathrm{CH})_{x}$ and trans- $(\mathrm{CH})_{x}$.

The cis/trans content was determined using IR and Raman Spectroscopy. The trans isomer was obtained from a fresh cisisomer sample kept at $200^{\circ} \mathrm{C}$ under vacuum during one hour.

\begin{tabular}{|c|c|c|c|c|}
\hline & \multirow{2}{*}{$\begin{array}{l}\text { cis/trans } \\
\text { Content }\end{array}$} & \multirow{2}{*}{$\frac{\begin{array}{c}\text { Number of } \\
\text { spins }\end{array}}{\mathrm{g}^{-1}}$} & \multirow{2}{*}{$\begin{array}{c}\Delta H_{\mathrm{pp}} \\
\text { Gauss }\end{array}$} & \multirow{2}{*}{$g$ Value } \\
\hline & & & & \\
\hline$c i s-(\mathrm{CH})_{x}$ & $\sim 95 \%$ & $\sim 10^{18}$ & $8.0 \pm 0.3$ & 2.0023 \\
\hline trans $-(\mathrm{CH})_{x}$ & $\sim 5 \%$ & $\begin{array}{l}\sim 10^{19} \\
-10^{20}\end{array}$ & $\begin{array}{c}1.25 \\
\pm 0.05\end{array}$ & 2.0023 \\
\hline
\end{tabular}


Magnetic Properties of Polyacetylene

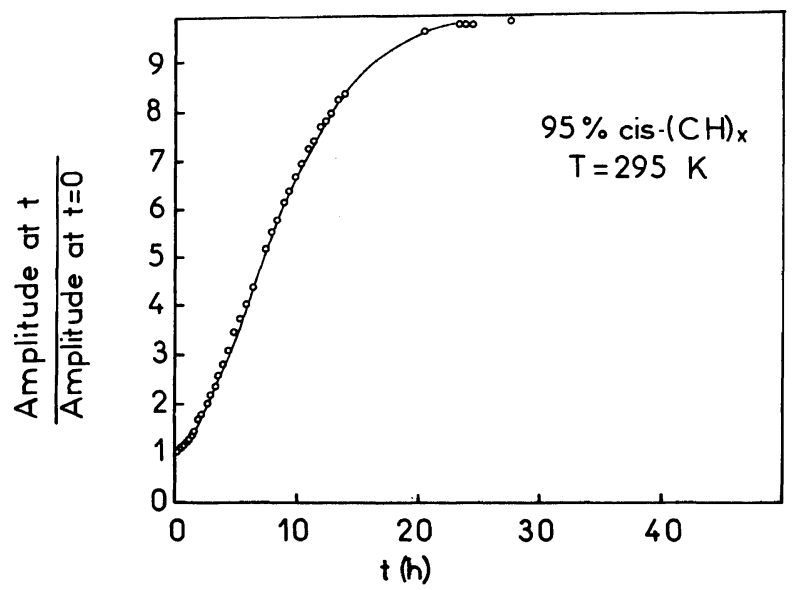

Figure 1. Time dependence of the ESR signal intensity of a $95 \%$ cis- $(\mathrm{CH})_{x}$ sample exposed to air at room temperature.

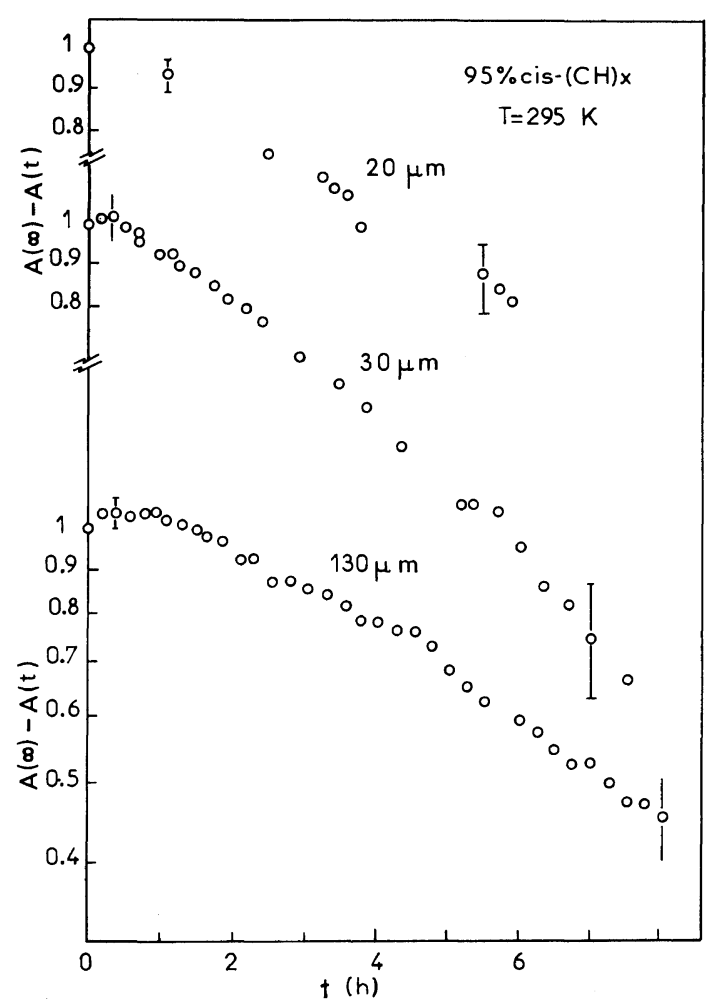

Figure 2. The difference between the ESR signal intensities at time $t$ and $\sim$ infinite time, normalized to unity for $t=0$, is plotted in a Log scale versus $t$, for three $95 \%$ cis- $(\mathrm{CH})_{x}$ samples having different thicknesses and submitted to air at room pressure and temperature. If not actually linear, the characteristic times of the observed dependences are quite similar in the three cases (see Table II).
With an increase in temperature, the signal coming from the catalytic system was strongly modified. At the same time, a small symmetric signal started growing at $g=2.0023$, the amplitude of which increased with increasing temperature. At room temperature this signal had an intensity corresponding roughly to $10^{18}$ spins per $\mathrm{g}$, a width (between the peaks of the absorption derivative) of 8.8 Gauss with a lorentzian shape (see Table I). Opening the tube to air at room temperature was shown to produce a great decrease in catalyst signal while the $(\mathrm{CH})_{x}$ signal continued to grow. We observed that the amplitude $A(t)$ of the spectra at time $t$ increases rapidly just after the opening to air and saturates to a constant value $A(\infty)$ after a few days (Figure 1) corresponding to an increase of approximately one order of magnitude in the number of unpaired electrons. On the other hand the line width $\Delta H_{\mathrm{pp}}$ does not change significantly at the time scale of three days.

The quantity $A(\infty)-A(t)$ represents the deviation from equilibrium of the ESR amplitude at time $t$. On Figure 2 we have plotted $A(\infty)-A(t)$ in log scale versus. $t$, for samples of various thicknesses. The observed variation is not actually linear. Nevertheless we can deduce a rough characteristic time of the order of 8 hours for the evolution of $A(t)$, which is observed to be independent on the sample thickness (Table II).

After more than three days at room air and temperature, the tubes containing the cis- $(\mathrm{CH})_{x}$ samples were sealed under vacuum and kept at 
Table II. Characteristic time of the evolution of the number of spins for a cis- $(\mathrm{CH})_{x}$ samples submitted to air at room temperature, with three different thicknesses

\begin{tabular}{cc}
\hline \multirow{2}{*}{ Thickness } & Characteristic time \\
\cline { 2 - 2 } & $\min$ \\
\hline $20 \mu \mathrm{m}$ & $480 \pm 90$ \\
\hline $30 \mu \mathrm{m}$ & $390 \pm 60$ \\
\hline $130 \mu \mathrm{m}$ & $480 \pm 60$ \\
\hline
\end{tabular}

$200^{\circ} \mathrm{C}$ for one hour in order to change the configuration from cis to trans. After that the ESR spectra showed a strong increase in intensity (between $\times 10$ and $\times 100$ depending on the starting value of the cis-isomer) while the linewidth $\Delta H_{\mathrm{pp}}$ decreased to a value of 2.5 Gauss. This value was obtained for various samples and seems to be a universal limit correlated with the type of treatment on the sample.

This value should be compared with the value $\Delta H_{\mathrm{pp}}=1.25$ Gauss for the trans isomer prepared from the $c i s$ isomer which had no contact with air.

\section{The trans-Isomer}

The trans configuration was obtained from the $c i s$ one by heating the sample for one hour as described above. The characteristics of the ESR spectra are summarized in Table I. We observed a strong increase in the unpaired-electrons density while the linewidth decreased. Kept under vacuum at room temperature, the characteristics of the spectra of these samples do not change with time.

If the sample is subjected to air at roomatmospheric pressure, we note change in the ESR spectra quite different from those observed on the cis isomer. The amplitude of the signal slightly decreases while its width increases up to 2.6 Gauss (Figure 3). A stationnary situation was attained after roughly two days. Nevertheless during the whole evolution the number of unpaired spins remained constant except for a slight decrease which occurred at the very beginning.

We must note that the stationary value for the linewidth obtained in this case is approximately the same as the one observed for a cis isomer first subjected to air and after being isomerized to the trans configuration. This suggests that the final stationary situation (oxidized trans configuration) obtained in the two cases does not depend on the manner to reach it.

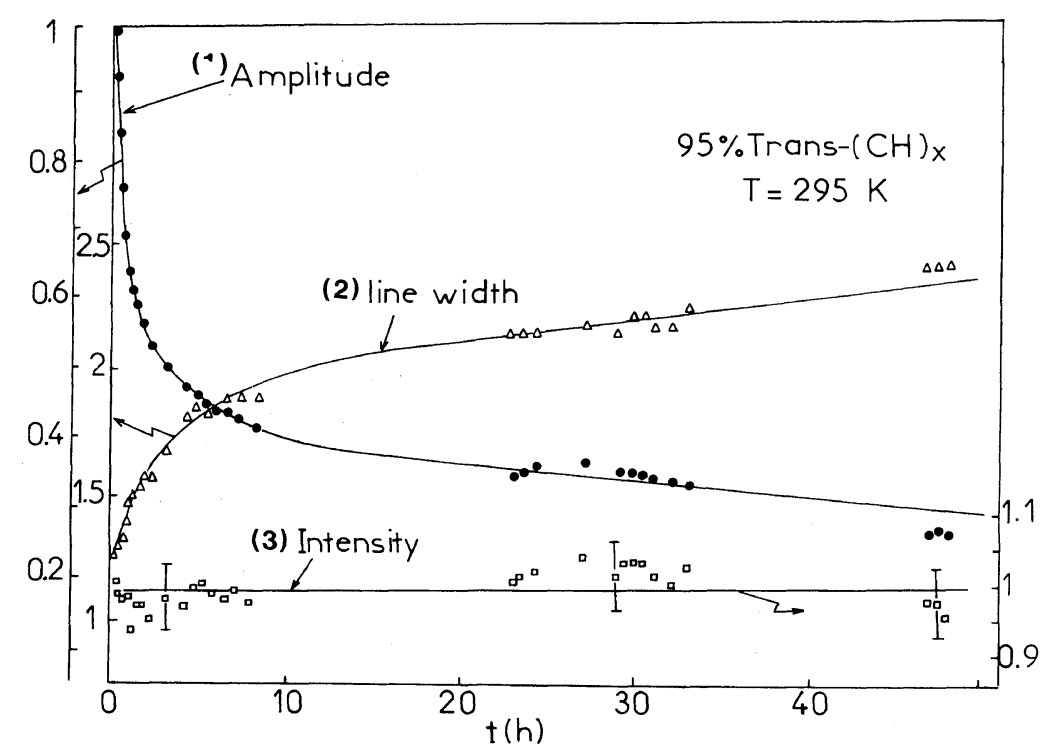

Figure 3. Time dependences of the ESR characteristics of a $95 \%$ trans- $(\mathrm{CH})_{x}$ sample exposed to air at room temperature: (1), amplitude normalized to unity for $t=0$; (2), linewidth in Gauss; (3), number of unpaired electrons normalized to unity for $t=0$. 
Magnetic Properties of Polyacetylene

a)<smiles>C=C/C=C\C=C/C=C/C=C\C=C\C=C/C=C</smiles>

b)

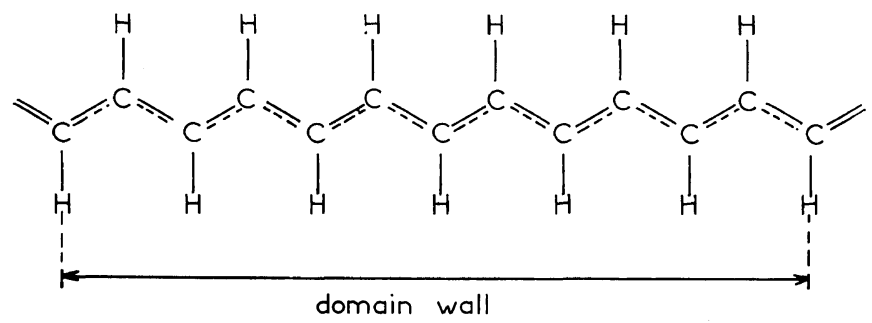

Figure 4. Neutral defect appearing in the trans configuration as a consequence of dephasing in the bond alternation: $a$, localized defect; $b$, delocalized defect spread over a given number of $C$ atoms, or domain wall.

\section{DISCUSSION}

The absence at low temperature of any signal on the $c i s$ isomer indicates that no neutral defects involving unpaired electrons exist in the case of cis configuration. This is compatible with the fact stated above that the two distinct geometries of this configuration are not energetically equivalent and this situation does not favour the existence of defects in the double-bond alternation. On the other hand the high density of unpaired electrons observed with the trans isomer is consistent with the existence of neutral defects of the type proposed by Chiang et $a l^{11}$ and Goldberg et al. ${ }^{6}$ (Figure 4), which is in agreement with the two energetically equivalent geometries of this isomer.

Between these two limiting cases, any intermediate situation, obtained as well by heating the $c i s$ isomer as by submitting it to air, must be understood in terms of partial isomerization.

The heating of the pure cis-isomer was shown ${ }^{13}$ to create unpaired electrons whose resonance $g$ value could not be distinguished from the free electron value.

The width of the observed spectra decreases when the trans content of the samples increases, as first shown by Shirakawa et al. ${ }^{16}$ Such a width has its origin partially in unresolved hyperfine interactions as stated by Snow et al. ${ }^{7}$ who compared the cases of $(\mathrm{CH})_{x}$ and $(\mathrm{CD})_{x}$ systems. We confirmed this conclusion, since spectra obtained on ${ }^{13} \mathrm{C}$ enriched samples have all show widths larger than the
Table III. Values of the linewidth $\Delta H_{\mathrm{pp}}$ of the ESR spectra obtained on the cis and trans isomer of the different undoped samples

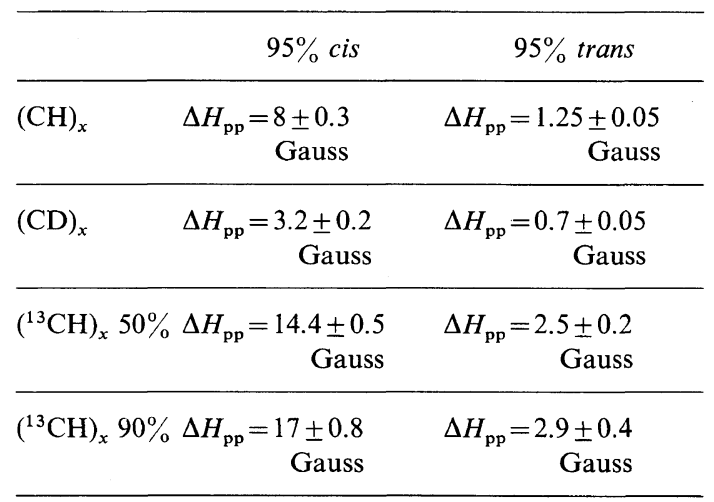

corresponding non enriched systems (Table III).

Weinberger et al. ${ }^{17}$ attempted to simulate numerically the ESR line for trans- $(\mathrm{CH})_{x}$ assuming that the width could be attributed entirely to unresolved hyperfine splitting, as a consequence of spin mobility. At $4.2 \mathrm{~K}$, a fit with their experimental data involved such a mobility over 30 to 40 carbon atoms along the chain. At room temperature, this mobility is expected to be considerably higher, as evidenced by the decrease in $\Delta H_{\mathrm{pp}}$ with increasing temperature. ${ }^{6}$ Therefore the larger width observed with a great cis content can be interpreted as follows. The trans content at room temperature for what we call our cis isomer is of the order of $5 \%$. This amount 
was isomerized quite rapidly when the sample heat from its polymerization temperature to room temperature. ${ }^{13}$ Consequently, we suggest such an amount to be located on chain ends where the ability to rotate around $\mathrm{C}-\mathrm{C}$ bonds is greater than in the middle of the chain. Using $n=1000$ as a mean value of the number of carbon atoms in one chain, as estimated by shirakawa et al. ${ }^{18}$ we then obtained trans sequences consisting of 25 carbon atoms. With the model proposed by Weinberger et al., ${ }^{17}$ unpaired electrons associated with trans sequences of such an extent would give an ESR spectrum width of $\sim 6$ Gauss which is compared reasonably with our experimental value of 8 Gauss (Table I), taking into account the crudeness of the assumptions model. This indicates that even at low temperature, a model assuming defects of relatively high mobility, which are intrinsic characteristics of the trans configuration, is consistent with the existing experimental data.

A study of the effect of air exposure allows us to gain further understanding of the magnetic properties of undoped samples. Snow et al. ${ }^{7}$ have studied the effect of oxygen on the characteristics of the ESR spectra. Nevertheless, a clear distinction between the cis and trans isomer cases was not made, leading to inaccurate conclusions concerning the observed behaviours. Goldberg et al. ${ }^{6}$ have also studied oxygen effects on the trans-isomer and have suggested the existence of a weak interaction of the chains with oxygen, followed by a slow chemical reaction. Our more detailed results obtained on the trans-isomer allow us to precise the following:

(i) The constant intensity of the ESR spectra during exposure to air indicates that no more unpaired electrons are induced by interaction with oxygen (Figure 4).

(ii) Nevertheless, an increase in linewidth (Figure 4) by a factor of $\sim 2$ is due to a reduction in spin mobility due to the presence of oxygen.

It is easy to understand these points if we suppose that oxygen interacts with the system as a diamagnetic $\mathrm{O}_{2}^{-2}$ species whose effect is only to create along the chains static-defects which limit the extent of mobility of the observed spins, leading to an increase in $\Delta H_{\mathrm{pp}}$. Such a chemical species, which can be considered as a partial cross-linking agent is likely in $(\mathrm{CH})_{x}$ as exposure to air is known to very rapidly damage its mechanical properties, ${ }^{19}$ and stretchable films become brittle.
Coming now to the cis-isomer case, the experimental results described above lead to the following conclusions.

(i) The presence of oxygen creates paramagnetic centers whose characteristics are the same as those of the starting unoxidized system.* ( $g$ value, linewidth and absence of hyperfine structure of the ESR spectra are similar before and after oxidation).

(ii) The constant value observed for the characteristic evolution time (Table II) of the signal intensity suggests that we are concerned with a diffusion process which does not depend on sample thicknesses.

This last point is consistent with the fibrous structure of $(\mathrm{CH})_{x}^{1}$ which allows a rapid diffusion inside the sample between the fibrils.

A further evolution is then due to diffusion in the fibrils themselves whose size does not depend on the sample thickness. This conclusion is in contradiction with a recent model proposed by Rice and Timonen ${ }^{20}$ who suggested that any doping species was more or less (depending on its molecular volume) restricted very near the surface of the fibrils. Nevertheless if interpreted in terms of molecular oxygen diffusion, the observed behaviour of Figure 3 would give a diffusion coefficient as low as $10^{-16} \mathrm{~cm}^{2} \mathrm{~s}^{-1}$. Even if we invoke the high crystallinity of the fibrils, ${ }^{21}$ which generally restricts diffusion, the above value seems very small compared with other types of semicrystalline polymers. ${ }^{22}$ Thus it seems reasonable to suppose that the effect of oxygen is to increase the rate at which the cis configuration transforms to the trans one. Diamagnetic $\mathrm{O}_{2}$ molecules by breaking double bonds along the chains, make rotation around $\mathrm{C}-\mathrm{C}$ bonds. The consecutive increase in trans content, which has been observed by infrared (IR) spectroscopy ${ }^{23}$ then appear as an increase in unpaired electrons. The fact that the linewidth does not change significantly during the intensity evolution indicates that the trans sequences created have an extent comparable with that obtained during the only thermal isomerization. This suggests a strong similitude between the effects of oxidation and thermal isomerization. This point is supported by the fact stated above that thermal isomerization fol-

* We must remind that the unoxidized cis system has at room temperature a cis content of $95 \%$ and that the ESR signal comes from the remainder $5 \%$ of trans isomer. 
lowed by oxidation leads to the same final situation (trans-isomer with $\Delta H_{\mathrm{pp}} \sim 2.5$ Gauss) than oxidation followed by thermal isomerization.

\section{CONCLUSION}

We have shown that the characteristics of the observed ESR spectra are governed by the trans content of the samples. Starting from the pure cisisomer, for which no ESR signal is observed, thermal isomerization causes unpaired electrons to appear. At a given temperature, the effect of oxidation increases during a certain time (a few days at room temperature) the rate at which isomerization proceeds. Additional experiments are needed for analyzing quantitatively the characteristics of the neutral defect which have been used for interpreting our experimental results.

Acknowledgments. The support for the ESR equipment (Bruker Minispec ER 10) by Bruker Spectrospin France is gratefully acknowledged. One of us (P.B.) is thankful to Pr. A. G. MacDiarmid for his very helpful discussions. Financial support from DRET under contract $\mathrm{n}^{\circ} 78 / 1259$ is gratefully acknowledged.

Note Added in Proof. Very recent results obtained by S. Lefrant et al. ${ }^{24}$ using Raman spectroscopy, clearly show that oxidation of a cis- $(\mathrm{CH})_{x}$ sample largely increase the rate (at room temperature) at which isomerization occurs, in agreement with our present conclusions.

\section{REFERENCES}

1. A. G. McDiarmid and A. J. Heeger, "Molecular Metals," Plenum Press, New York, N.Y., 1979, p 161.

2. (a) P. Bernier, M. Rolland, M. Galtier, A. Montaner, M. Regis, M. Candille, C. Benoit, M. Aldissi, C. Linaya, F. Schué, J. Sledz, J. M. Fabre, and L. Giral, J. Phys. Lett., 40, L-297 (1979). (b) M. Rolland, P.
Bernier, M. Disi, C. Linaya, J. Sledz, F. Shué, J. M. Fabre, and L. Giral, J. Phys. Lett., 41, L-165 (1980).

3. G. Mihaly, G. Vancso, S. Pekker, and A. Janossy, submitted to Synth. Met.

4. A. G. McDiarmid, unpublished results.

5. D. J. Berets and D. S. Smith, Trans. Faraday Soc., 64, 823 (1968).

6. I. B. Goldberg, H. R. Crowe, P. R. Newman, A. J. Heeger, and A. G. McDiarmid, J. Chem. Phys., 70, 1132 (1979).

7. A. Snow, P. Brant, D. Weber, and N. L. Yang, J. Polym. Sci., Polym. Lett. Ed., 17, 263 (1979).

8. W. P. Su, J. R. Schrieffer, and A. J. Heeger, Phys. Rev. Lett., 42, 1698 (1979).

9. T. Yamabe, K. Tanaka, H. Teramae, K. Fukui, A. Imamura, H. Shirakawa, and S. Ikeda, Solid State Commun., 29, 329 (1979).

10. J. L. Bredas and J. Delhalle, to be published.

11. C. K. Chiang, Y. W. Park, A. J. Heeger, H. Shirakawa, E. J. Louis, S. C. Gau, and A. G. McDiarmid, Phys. Rev. Lett., 39, 1098 (1977).

12. T. Ito, H. Shirakawa, and S. Ikeda, J. Polym. Sci., Polym. Chem. Ed., 12,11 (1974); 13, 1943 (1975).

13. P. Bernier, M. Rolland, C. Linaya, M. Disi, Polymer, 21, 7 (1980).

14. H. Hirai, H. Katsuma, N. Isamu, and S. Makishima, J. Polym. Sci., A-1, 8, 147 (1970).

15. M. Takeda, K. Iimura, Y. Nozawa, M. Hisatome, and N. Koide, J. Polym. Sci., C, No. 23, 741 (1968).

16. H. Shirakawa, T. Ito, and S. Ikeda, Makromol. Chem., 179, 1565 (1978).

17. B. R. Weinberger, J. Kaufer, A. J. Heeger, and A. G. McDiarmid, to be published.

18. H. Shirakawa, M. Sato, A. Kamano, S. Kawabami, K. Soga, and S. Ikeda, to be published.

19. A. G. McDiarmid and A. J. Heeger, unpublished results.

20. M. J. Rice and J. Timonen, Phys. Lett., 73A, 368 (1979).

21. H. Shirakawa and S. Ikeda, to be published.

22. D. W. Van Krevelen, "Properties of Polymers," Elsevier Publishers, Amsterdam, 1976.

23. M. Galtier and A. Montaner, private communication.

24. S. Lefrant, E. Rzepka, P. Bernier, M. Rolland, and M. Disi, Polymer (1980), to be published. 